\title{
Utilización de modelo de trabajo pasivo en la confección de prótesis fijas atornilladas de arco completo sobre implantes. Reporte de caso
}

Using a passive master model for the preparation of implant-supported full-arch prosthesis. A case report

Enrique Castro Aguilar ${ }^{1, a}$, Carlos Matta Morales ${ }^{2, a, c, d}$, Elías Miguel Rivera Álvarez ${ }^{3, a}$, Andrea Camere Colarossi ${ }^{2, b}$

\section{RESUMEN}

El procedimiento para la elaboración de una prótesis atornillada de arco completo sobre implantes (PAACI), requiere como requisito mecánico fundamental un acoplamiento y ajuste pasivo para garantizar un adecuado comportamiento biomecánico de dicha prótesis a largo plazo. El proceso de confección de una infraestructura para una PAACI que brinde una pasividad adecuada es minucioso y requiere el uso de materiales de alta precisión, los procedimientos clínicos y de laboratorio deben ser cuidadosos para minimizar las deficiencias de éstos y llevar a buen término el trabajo final. El presente artículo muestra un reporte de caso donde se plantea la utilización de un modelo de trabajo pasivo mediante la verificación intraoral para corregir las limitaciones de los materiales utilizados y reducir los procedimientos clínicos y de laboratorio.

PALABRAS CLAVE: Prótesis dental de soporte implantado, ajuste de precisión de prótesis, implantes dentales. (DeCS, BIREME)

Cirujano Dentista egresado de la Facultad de Odontología, Universidad Nacional de la Amazonía Peruana. Iquitos, Perú.

Facultad de Estomatología Roberto Beltrán, Universidad Peruana Cayetano Heredia. Lima, Perú.

Facultad de Odontología, Universidad de Maryland. Washington DC, Estados Unidos.

Especialista en Rehabilitación Oral

Especialista en Periodoncia e Implantes.

Magister en Estomatología.

Profesor. 


\section{SUMMARY}

The use of a verification jig and a passive master model for the preparation of implant-supported full-arch prosthesis: a case report.

In order to achieve long term prognosis, the fabrication of a implant-supported full-arch prosthesis (ISFAP) should follow principles of mechanical coupling and passive fitting. This is achieved through the use of highprecision techniques and materials for both chair side and laboratory to minimize shortcomings and get an adequate result. This article reports the use of a verification jig to confirm passive fit. The passive working model is tried intraorally to correct for possible errors, and thereby reduces chair time and the need to send the case out for numerous laboratory corrections.

\section{KEYWORDS: Implant-supported dental prosthesis, accuracy prosthesis, dental implants. (MeSH, NLM)}

\section{INTRODUCCIÓN}

Las prótesis fijas atornilladas de arco completo sobre implantes (PAACI) llamadas también prótesis hibridas, desde que fueron propuestas inicialmente por el doctor Brannemark hasta la actualidad, han demostrado una alta tasa de éxito a largo plazo (1-5).

Las ventajas que presentan este tipo de prótesis son: a) recuperan la función y estética de los tejidos perdidos, b) preservan y mantienen los tejidos remanentes, c) posibilidad del retiro periódico de la prótesis para la evaluación clínica de los tejidos peri-implantarios e higiene, d) reversibilidad por si se requiere la reparación de la prótesis, e) ausencia del agente cementante, lo que permite suprimir la posible contaminación de las zonas peri-implantarias con el cemento (6-10). Todas éstas son importantes ventajas, necesarias sobre todo por tratarse de una prótesis de arco completo; sin embargo, está propensa a problemas biomecánicos. Así, Papaspyridakos y col. (11) realizaron una revisión sistemática donde evaluaron estudios prospectivos de cinco y diez años de seguimiento, encontrando, problemas técnicos (fractura o pérdida del tornillo de la prótesis, fractura o desgaste del acrílico de revestimiento de la prótesis, fractura de la infraestructura de la prótesis) en un $10,4 \%$ a los 5 años y de $20,8 \%$ a los 10 años de los casos, y problemas biológicos (hipertrofia, hiperplasia e inflamación de los tejidos peri-implantarios) en un $13 \%$ a los 5 años y $26 \%$ a los 10 años. Estos son valiosos datos estadísticos, que nos sugieren una conducta precavida de índole técnico así como también la obligación de una inspección exhaustiva de los tejidos que rodean los implantes.

Existen requisitos biológicos y mecánicos que se deben cumplir al confeccionar una PAACI. Los requisitos bilógicos son: rehabilitar los tejidos duros y blandos ausentes permitiendo la preservación de los tejidos remanentes, así como la accesibilidad a los instrumentos de higiene para garantizar la salud periimplantaria. Mientras que los requisitos mecánicos son: retención, estabilidad, pasividad, solidez estructural, extensión adecuada de la prótesis y una oclusión funcional (12-13).

La pasividad es el requisito mecánico que resulta un desafío para el odontólogo. Diversos estudios (13-15) indican que la tensión entre los implantes y las PAACI está asociada a la pérdida ósea a nivel crestal y el aflojamiento o la fractura de los tornillos que sujetan la prótesis. El objetivo de la pasividad es evitar dicha tensión mediante un ajuste pasivo con la menor desadaptación posible entre el pilar y la prótesis (16). El rango de desadaptación aceptable, inicialmente descrito por Brannemark (16), estaba entre los valores menores a $10 \mu \mathrm{m}$. Sin embargo, estudios recientes como el realizado por Jemt (17), han demostrado que desadaptaciones menores a 150 $\mu \mathrm{m}$ no causarían ninguna complicación clínica a largo plazo, pudiéndose considerar como aceptable.

El proceso de confección de la infraestructura para una PAACI que brinde una pasividad adecuada es minucioso y requiere el uso de materiales de alta precisión, los procedimientos clínicos y de laboratorio deben ser cuidadosos para minimizar las deficiencias de estos y llevar a buen término el trabajo final. Estas deficiencias son: a) los materiales de transferencia de la posición de los implantes presentan contracción de polimerización que varía de 0,11 a $0,45 \%$, b) los yesos de vaciado sufren una expansión de endurecimiento de $0,9 \%, \mathrm{y}$ c) la contracción del metal colado mediante la técnica de la cera perdida, varía según la extensión de la infraestructura, 
normalmente se espera una contracción del 1,5\% en metales preciosos, porcentaje que se puede duplicar si se trata de aleaciones semipreciosas $(18,19)$. Siendo ésta última, una deficiencia superada a través de la confección de la infraestructura mediante el mecanizado industrial o la soldadura laser directa de barras de titanio prefabricadas (21).

Para superar las deficiencias mencionadas anteriormente, se plantea la técnica de la utilización de modelo de trabajo pasivo para la elaboración de PAACI mediante la verificación intraoral.

\section{REPORTE DE CASO}

Paciente de sexo masculino de 45 años de edad, con aparente buen estado general, ausencia de alteraciones sistémicas y sin antecedentes médicos relevantes, ingresó a la Clínica Dental de la Universidad Peruana Cayetano Heredia presentando edentulismo del maxilar superior. Fue manejado por los Servicios de Periodoncia e Implantes y Rehabilitación Oral para restablecer las funciones del sistema estomatognático a través de la colocación de prótesis fija de arco completo sobre seis implantes.

Posterior a la osteointegración exitosa de dichos implantes y la colocación de los pilares cónicos, se inició la elaboración de la parte protésica de una PAACI; procedimientos que incluyen: a) transferencia de los implantes/pilares, b) elaboración de la infraestructura metálica, c) verificación del ajuste pasivo de la infraestructura metálica, d) prueba de enfilado de los dientes, e) instalación y controles de la prótesis final.

La técnica de utilización del modelo pasivo plantea una variación en los tres primeros pasos del procedimiento protésico tradicional, los cuales serán descritos en el presente artículo.

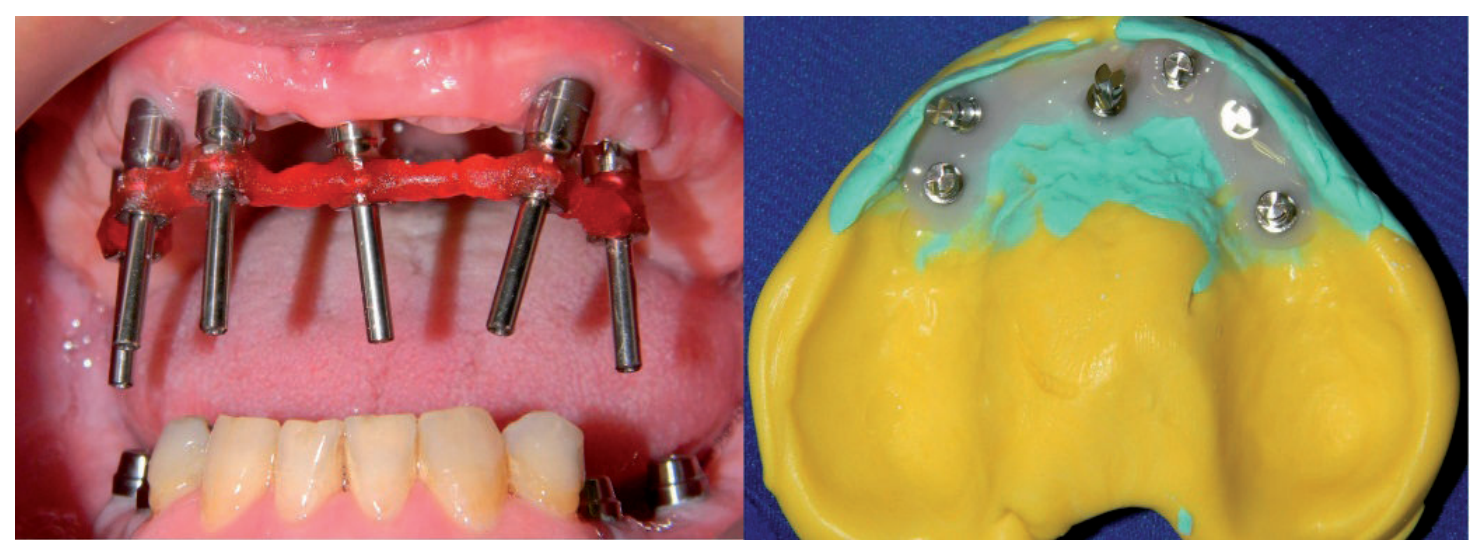

Figura 1. Transferencia de los implantes y reproducción de encía falsa.

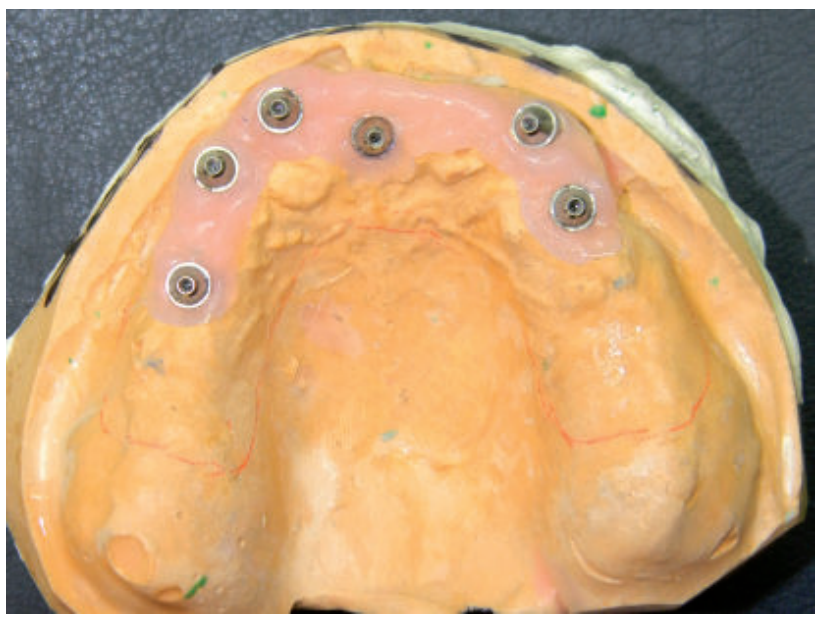

Figura 2. Modelo maestro. 


\section{Elaboración de la técnica del modelo pasivo}

Se realiza la transferencia de los implantes a través de la ferulización de los transferentes con resina acrílica de combustión completa de auto polimerización (Pattern Resin ${ }^{\mathrm{TM}}$ LS, GC América) posteriormente se procede al arrastre de los transferentes con silicona por adición de consistencia pesada y fluida (Panasil® Putty/Initial contact light, Kettenbach $\mathrm{GmbH} \& \mathrm{Co}$. KG) mediante la utilización de una cubeta abierta personalizada (Figura 1).

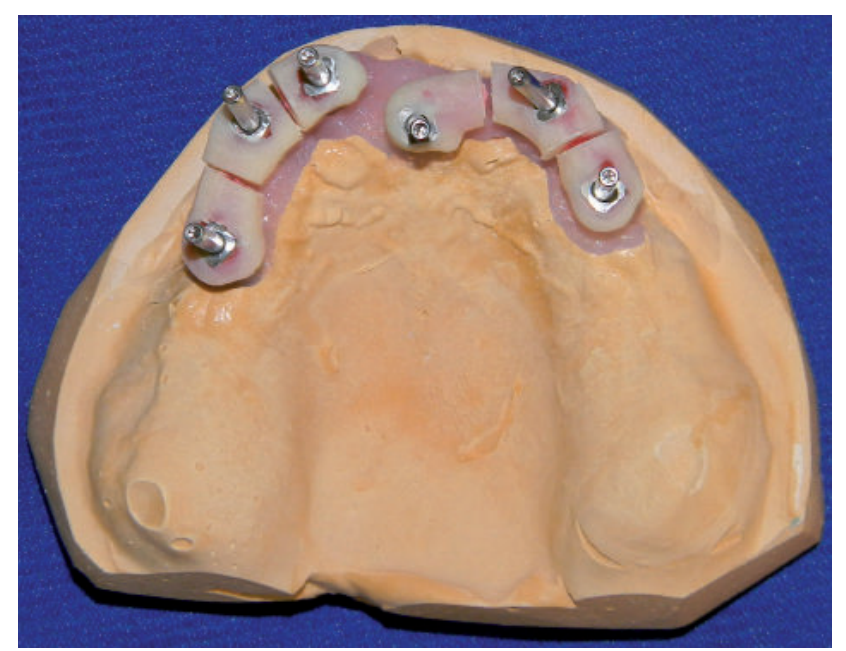

Figura 3. Jig de verificación seccionado.
Luego se procede al vaciado del molde con silicona de laboratorio para la reproducción de la encía (Gingifast Elastic ${ }^{\mathrm{TM}}$ Zhermack) y yeso tipo IV (Elite Rock $^{\mathrm{TM}}$ Zhermack) siguiendo las recomendaciones del fabricante (Figura 2).

Pasado el tiempo del fraguado final del modelo de trabajo, se realiza en el laboratorio la elaboración de un dispositivo de resina acrílica llamado "jig de verificación" sobre los transferentes, el cual es seccionado para la comprobación clínica de la exactitud del modelo de trabajo (Figura 3).

La evaluación de la precisión de la transferencia se realizó uniendo las partes seccionadas del jig de verificación intraoralmente mediante el empleo de resina acrílica de combustión completa de auto polimerización (Pattern Resin ${ }^{\mathrm{TM}}$ LS, GC América). Luego en el laboratorio se realizó una meticulosa verificación visual en el modelo maestro y el uso de la prueba del tornillo "one-screw test" propuesta por Jemt (17) (Figura 4).

En caso que existiese una variación de la posición tridimensional de los análogos del modelo de trabajo se procede al retiro del análogo o los análogos involucrados en la distorsión, seguidamente se realiza

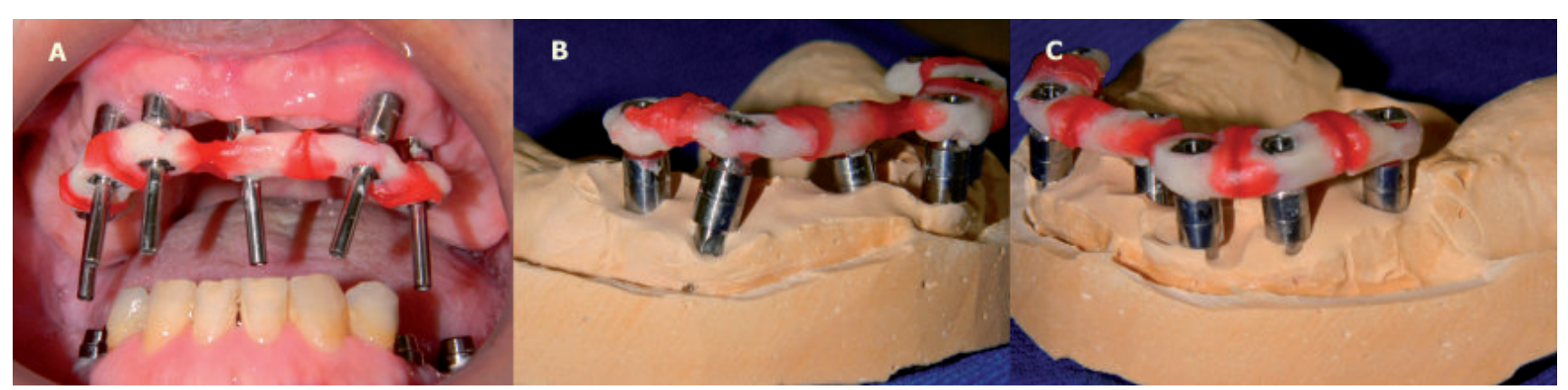

Figura 4. A: Ferulización intraoral del jig de verificación. B y C: Comprobación de la pasividad en el modelo maestro.

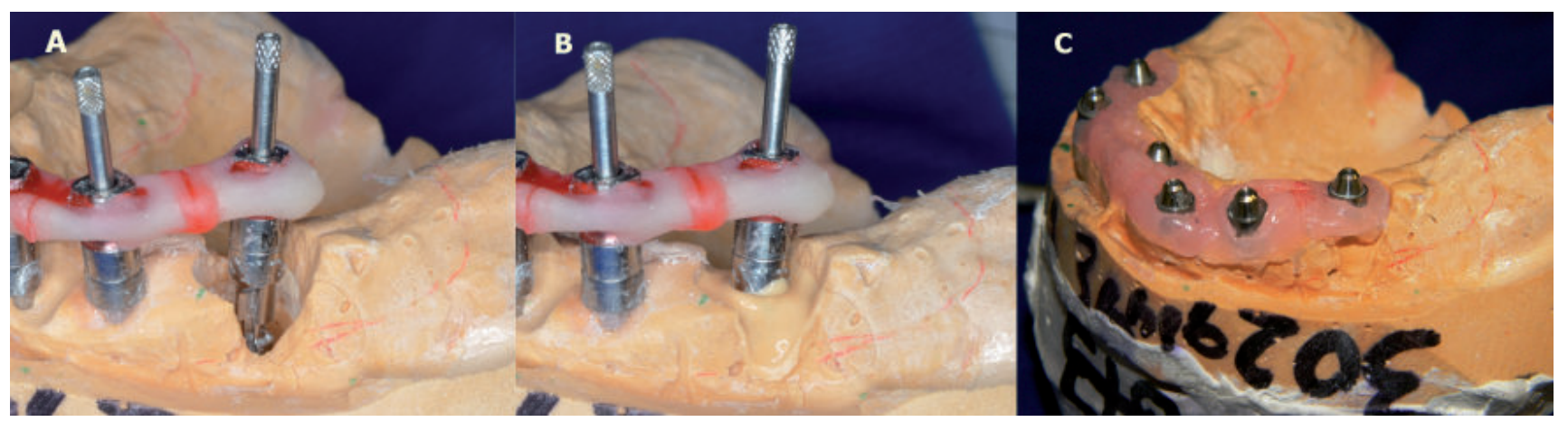

Figura 5. A: Recolocación del análogo en la posición correcta utilizando el jig de verificación.

B: Vaciado selectivo del análogo comprometido. C: Recolocación de la falsa encía. 
el reajuste y vaciado del mismo empleando el jig de verificación para la correcta posición de la situación clínica (Figura 5). La determinación del análogo o análogos a ser reposicionados se realiza a través de la prueba del tornillo "one screw test".

La elaboración de la infraestructura metálica de la PAACI se puede realizar a través de la técnica de colado de aleaciones nobles o no nobles, mecanizado industrial de titanio o la soldadura laser directa de barras de titanio prefabricadas (22).

Cuando se realiza la técnica de colado de las aleaciones metálicas, en la mayoría de los casos, es necesario el empleo de la soldadura de dos o más elementos seccionados de la infraestructura para compensar la contracción del metal que puede ir de $1,5 \%$ a 3\% dependiendo la calidad de la aleación (20) para lo cual se determina la fabricación seccionada de la infraestructura para su posterior soldaje.
La técnica de modelo de trabajo pasivo puede prescindir de la realización de la unión de las partes de la infraestructura metálica seccionadas clínicamente en la boca del paciente (Figura 6) puesto que la exactitud la posición de los análogos en el modelo de trabajo ya fue comprobada anteriormente permitiendo la soldadura de la infraestructura guiándose de la pasividad en el laboratorio, ahorrándose de esta manera 2 o más citas (Figura 7).

Concluida la fabricación de la infraestructura metálica se procede a la verificación clínica de la pasividad para finalmente elaborar la supraestructura de dientes y falsa encía de la PAACI (Figura 8).

Otras técnicas de confección de las infraestructuras metálicas son: el mecanizado industrial de titanio y la soldadura laser directa de barras de titanio prefabricadas. Estas técnicas eliminan la contracción del metal durante su fabricación lo que permite excluir

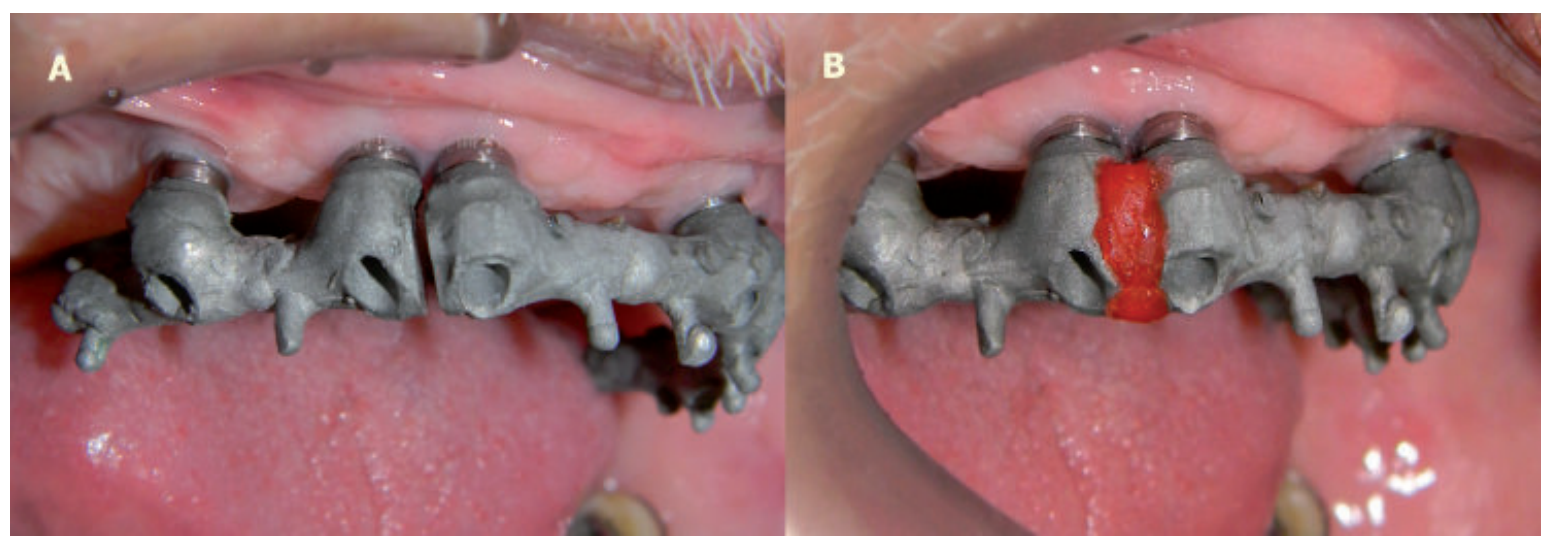

Figura 6. A: Colocación intraoral de la infraestructura seccionada para la unión en ajuste pasivo. B: Soldadura en frío con acrílico de combustión completa.

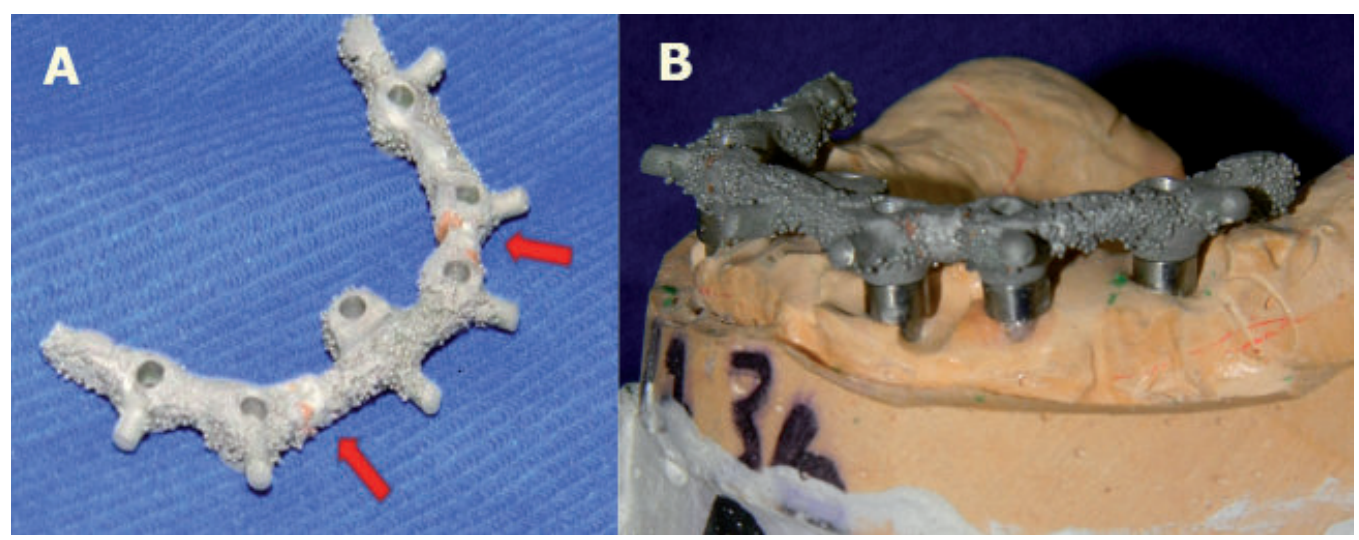

Figura 7. A: Infraestructura corregida en el laboratorio sin necesidad de soldadura en frio, gracias al uso del modelo pasivo. B: Verificación de la pasividad en el modelo maestro. 


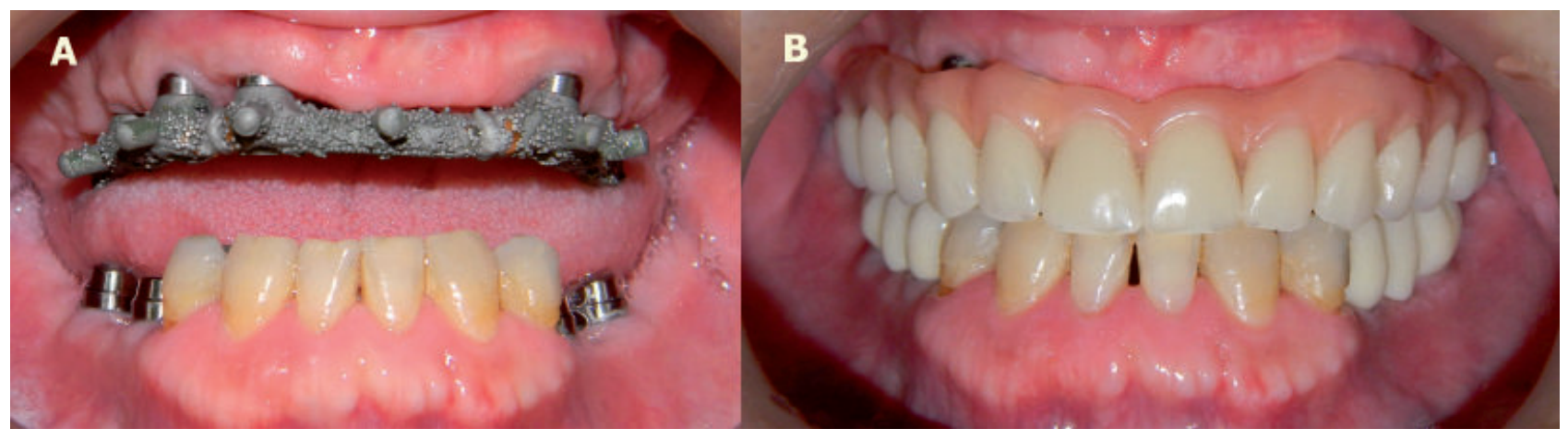

Figura 8. A: Verificación de la pasividad clínica final. B: PFAAC finalizada.

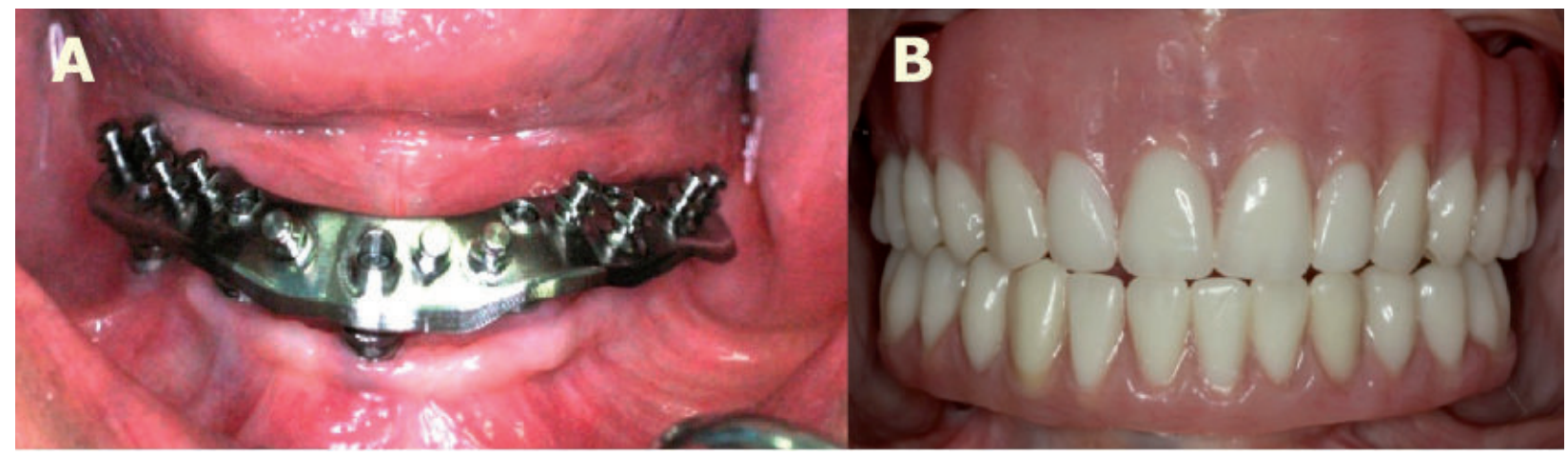

Figura 9. A: Infraestructura de la PFAAC de titanio confeccionada mediante el diseño por ordenador y mecanizado industrial CAD/CAM. B: PFAAC finalizada.

el uso de la soldadura de elementos seccionados de la misma. Esta ventaja importante será factible siempre que la exactitud del modelo de trabajo lo permita haciendo imprescindible la verificación de la pasividad mediante la utilización de la técnica descrita (Figura 9).

\section{DISCUSIÓN}

En concordancia con algunos autores $(21,22)$ no es posible la verificación objetiva ni la confección de las PAACI con una adaptación pasiva exacta, incluso las técnicas sofisticadas de diseño y manufactura asistidas por ordenador (CAD/CAM) no pueden ofrecer una pasividad verdadera, siendo para el clínico un desafío la tarea de estimar con criterio minucioso este requisito que le posibilite estar dentro de los rangos de desadaptación aceptables (menor a $150 \mu \mathrm{m})$.

Los procedimientos de transferencia de la posición de los implantes y pilares al modelo de trabajo mencionados en el presente artículo corresponden al protocolo de la mayoría de clínicos actualmente y la técnica de utilización de modelo de trabajo pasivo para la confección de PAACI trata de ser una propuesta elemental para corregir las limitaciones de los materiales utilizados y los procedimientos de laboratorio. Sin embargo, actualmente se están desarrollando tecnologías sofisticadas para la transferencia de escaneo intraoral digital, confección por ordenador y mecanizado industrial CAD/CAM para mejorar dicho protocolo y ofrecer una técnica precisa y rápida (23).

La tendencia actual del uso del titanio en las PAACI por sus propiedades de biocompatibilidad, bajo peso molecular, alta resistencia a la corrosión y adecuada resistencia mecánica (24), es un requisito cada vez más solicitado por los clínicos siendo una alternativa factible gracias al mecanizado industrial o la soldadura laser directa de barras de titanio prefabricadas cuya elaboración requieren de una transferencia exacta para posibilitar la confección de una infraestructura que no precise de modificaciones posteriores a su fabricación (25).

Torsello y col. (25) en un estudio comparativo encontraron diferencias en las distintas técnicas de elaboración de infraestructuras, observándose valores 
de desadaptación entre $78 \mu \mathrm{m}( \pm 48)$ para la técnica del colado de metal, $33 \mu \mathrm{m}( \pm 19)$ para la soldadura laser directa de barras de titanio prefabricadas y 27 $\mu \mathrm{m}( \pm 15)$ para el mecanizado industrial, siempre que se realice una técnica de transferencia adecuada.

Finalmente, se puede concluir que la transferencia de la posición tridimensional de los implantes y la confección de las PAACI presentan limitaciones que impiden la realización del cumplimiento de los requisitos mecánicos; y consecuentemente, necesitan ser identificadas y compensadas, para que se optimice el resultado final.

\section{Correspondencia:}

Enrique Gabriel Castro Aguilar

Calle Los Capulíes No 757, Dpto. 302, Urb. La

Aurora - Miraflores. Lima, Perú.

Correo electrónico: castroaguilarenrique@gmail.com

\section{REFERENCIAS BIBLIOGRÁFICAS}

1. Albrektsson T, Brånemark PI, Hansson HA, Lindström J. Osseointegrated titanium implants. Requirements for ensuring a long-lasting, direct bone-to-implant anchorage in man. Acta Orthop Scand. 1981; 52(2):15570.

2. Bergkvis G, Sahlholm S, Nilner K, Lindh C. Implantsupported fixed prostheses in the edentulous maxilla. A 2-year clinical and radiological follow-up of treatment with non-submerged ITI implants. Clin Oral Implants Res. 2004; 15(3):351-9.

3. Rasmusson L, Roos J, Bystedt H. A 10-year follow-up study of titanium dioxide-blasted implants. Clin Implant Dent Relat Res. 2005; 7(1):36-42.

4. Fischer K, Stenberg T, Hedin M, Sennerby L. Five-year results from a randomized, controlled trial on early and delayed loading of implants supporting full-arch prosthesis in the edentulous maxilla. Clin Oral Implants Res. 2008; 19(5):433-41.

5. Gallucci GO, Morton D, Weber HP. Loading protocols for dental implants in edentulous patients. Int J Oral Maxillofac Implants. 2009; (24):132-46.

6. Vigolo P, Givani A, Majzoub Z. Cemented versus screw-retained implant-supported single-tooth crowns: a 4-year prospective clinical study. Int J Oral Maxillofac Implants. 2004; 19(2):260-5.

7. Shadid R, Sadaqa NA. Comparison between screw- and cement-retained implant prostheses. A literature review. J Oral Implantol. 2012; 38(3):298-307.

8. Chee W, Felton DA, Johnson PF, Sullivan DY. Cemented versus screw-retained implant prostheses: which is better? Int J Oral Maxillofac Implants. 1999; 14(1):13741.

9. Michalakis KX, Hirayama H, Garefis PD. Cement retained versus screw-retained implant restorations: a critical review. Int J Oral Maxillofac Implants. 2003; 18(5):719-28.

10. Hebel KS, Gajjar RC. Cement-retained versus screwretained implant restoration: achieving optimal occlusion and esthetics in implant dentistry. J Prosthet Dent. 1997; 77(1):28-35.

11. Papaspyridakos P, Chen CJ, Chuang SK, Weber HP, Gallucci GO. A systematic review of biologic and technical complications with fixed implant rehabilitations for edentulous patients. Int $\mathrm{J}$ Oral Maxillofac Implants. 2012; 27(1):102-10.

12. Serino G, Ström C. Peri-implantitis in partially edentulous patients: association with inadequate plaque control. Clin Oral Implants Res. 2009; 20(2):169-74.

13. Karl M, Rosch S, Graef F. Static implant loading caused by as-cast metal and ceramic veneered superstructures. J Prosthet Dent. 2005; 93(4):324-30.

14. De Sousa SA, de Arruda Nobilo MA, Henriques GE, Mesquita MF. Passive fit of frameworks in titanium and palladium-silver alloy submitted the laser welding. J Oral Rehabil. 2008; 35(2):123-7.

15. McGlumphy EA, Mendel DA, Holloway JA. Implant screw mechanics. Dent Clin North Am. 1998; 42(1):7189.

16. Branemark PI. Osseointegration and its experimental background. J Prosthet Dent. 1983; 50(3):399-410.

17. Jemt T. Failures and complications in 391 consecutively inserted fixed prostheses supported by Branemark implants in edentulous jaws: a study of treatment from the time of prosthesis placement to the first annual checkup. Int J Oral Maxillofac Implants. 1991; 6(3):270-6.

18. Pegoraro LF. Prótesis Fija. 1ra ed. Sao Paulo: Artes Médicas Latinoamérica; 2001.

19. Misch CE. Prótesis dentales sobre implantes. 1ra ed. Madrid: Elsevier; 2006.

20. Bryant RA, Nicholls JI. Measurement of distortions in fixed partial dentures resulting from degassing. J Prosthet Dent. 1979; 42(5):515-20.

21. Kan JY, Rungcharassaeng K, Bohsali K, Goodacre CJ, Lang BR. Clinical methods for evaluating implant framework fit. J Prosthet Dent. 1999; 81(1):7-13.

22. Karl M, Taylor TD. Effect of material selection on the passivity of fit of implant-supported restorations created with computer-aided design/computer-assisted manufacture. Int J Oral Maxillofac Implants. 2011; 26(4):739-45.

23. Karl M, Graef F, Schubinski P, Taylor T. Effect of intraoral scanning on the passivity of fit of implantsupported fixed dental prostheses. Quintessence Int. 2012; 43(7):555-62. 
24. Boeckler AF, Lee H, Psoch A, Setz JM. Prospective observation of $\mathrm{CAD} / \mathrm{CAM}$ titanium-ceramic-fixed partial dentures: 3-year follow-up. J Prosthodont. 2010; 19(8):592-7.

25. Torsello F, di Torresanto, VM, Ercoli C, Cordaro L. Evaluation of the marginal precision of one-piece complete arch titanium frameworks fabricated using five different methods for implant-supported restorations. Clin Oral Implants Res. 2008; 19(8):772-9.

Recibido: 21/11/2013 Aceptado: 09/02/2014 